\title{
Observation of magnetic nanoparticles ring formation and dynamics with a fast video camera
}

\author{
M. Bacia, ${ }^{* 1}$ J. Masajada, $^{1}$ and S. Drobczyński ${ }^{1}$ \\ ${ }^{1}$ Institute of Physics, Wroclaw University of Technology, Wyspianskiego 27, 50-370 Wroclaw, Poland
}

Received October 29, 2013; accepted December 3, 2013; published December 31, 2013

\begin{abstract}
Holographic optical tweezers were used to show the interaction between a strongly focused laser beam and magnetic nanoparticles in a ferrofluid. When the light intensity was high enough, magnetic nanoparticles were removed from the beam center, forming a dark ring. This behavior was observed in slow motion. The creation of dark rings is a very dynamic process which was further described in the following paper.
\end{abstract}

Ferrofluids are colloidal suspensions of magnetic particles having a size of about $10 \mathrm{~nm}$, dispersed in a liquid (organic or water) [1-2]. The magnetic particles are coated with a surfactant to inhibit their aggregation and sedimentation. Ferrofluids have both liquid and magnetic properties which are useful for various applications.

Optical tweezers are used for optical manipulation of micro and nano objects [3-4]. Holographic optical tweezers [5] allow for the creation of few tens of optical traps within the area of a tightly focused laser beam. The traps can be moved independently in the $x-y-z$ direction. Different kinds of traps (light, dark [6], Bessel [7]) can be generated simultaneously. Figure 1 shows the scheme of the holographic optical tweezers made in our laboratory. It is based on an inverted biological microscope Olympus IX71. A spatial light modulator Holoeye-Pluto was used for optical traps generation. We have used: a laser diode $980 \mathrm{~nm}$ with the output beam power up to $300 \mathrm{~mW}$. We have used the beam power up to $70-100 \mathrm{~mW}$ (measured before the microscope objective lens). The beam was focused with an immersion objective Olympus UPlanFL N 100x/1.30 Oil with NA=1.3. The focused spot had a radius of $\sim 2 \mu \mathrm{m}$. The images of the sample were taken under white light illumination. We used a halogen projection lamp made by Philips (type 7023).

\footnotetext{
${ }^{*}$ E-mail: marcin.bacia@pwr.wroc.pl
}

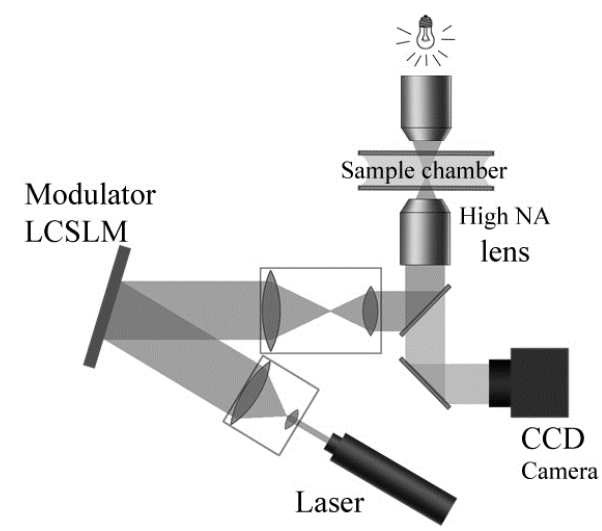

Fig. 1. Optical tweezers scheme. Optical traps were created and controlled by a spatial light modulator (LCSLM). Images were taken by camera under white-light illumination.

Optical tweezers have been already used for nanofluids investigations (see [8] for references). The light action on ferrofluids was also described in [1], [9-11]. In [1] the reader can find a review of optical properties of ferrofluids. In paper [12] we reported on the new phenomenon discovered when investigating ferrofluids with holographic optical tweezers. The advantages of a holographic system are high energy density and the possibility to work with many different optical traps at the same time. We showed that when light energy density is strong enough magnetic nanoparticles are organized into a ring. The central part of the ring becomes transparent (the ferrofluid transparency is low). In multitrap mode each trap generates its own ring and these rings interact with each other in a complicated way. In this work we used a fast video camera to record rings behavior. We worked with the same ferrofluid as previously (supplied by Webcraft $\mathrm{GmbH}$ ). The magnetic particle size was about $10 \mathrm{~nm}$. The volume fraction of the magnetic particles was $\sim 5 \%$ (supplier data). No external magnetic field was applied in our experiments. The thickness of measured samples was about $6 \pm 4 \mu \mathrm{m}(2 \mu \mathrm{l}$ of ferrofluid spread under $18 \times 18 \mathrm{~mm}$ cover slide). 
We have observed the process of dark ring formation using a fast camera (Microtron Inspecta MC1362) with a frame rate of 3000fps. The ring formation appears to be very fast, because it cannot be observed even with the above mentioned frame rate. The time separation between frames was about $330 \mu$ s and still the whole process was taking place while the two successive frames were captured (Fig. 2). We could distinguish three phases of the ring creation process:

1. The flash: In this phase the ring is created. It takes $\leq$ $330 \mu$ s and has not been precisely observed.
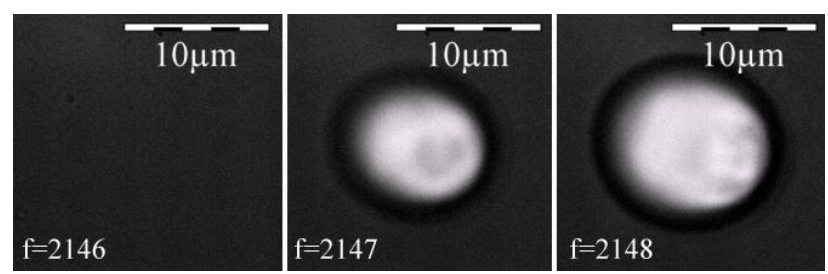

Fig. 2. Three successive frames from a movie showing flash ring formation ( $\mathrm{f}$ on the picture is frame number)

(see also movie file 1- ring creation in slow motion - $100 \times$ slowed).

2. After flash relaxation: It lasts about $10 \mathrm{~ms}$. In this stage, the dark ring shrinks down, up to $50 \%$ of its initial size. At the beginning of this phase the bright "tails" connected to the ring are barely visible and they start from several places on the ring (we have observed up to 6 tails). In most cases they are "closed" - two tails are connected, forming a closed line. At the end of this step, there are usually only 1 to 4 bright tails (Fig. 3). In most cases, at least one of them is "open" (forms a nearly straight line, which is not connected with other tails within the observation area) (Fig. 4).
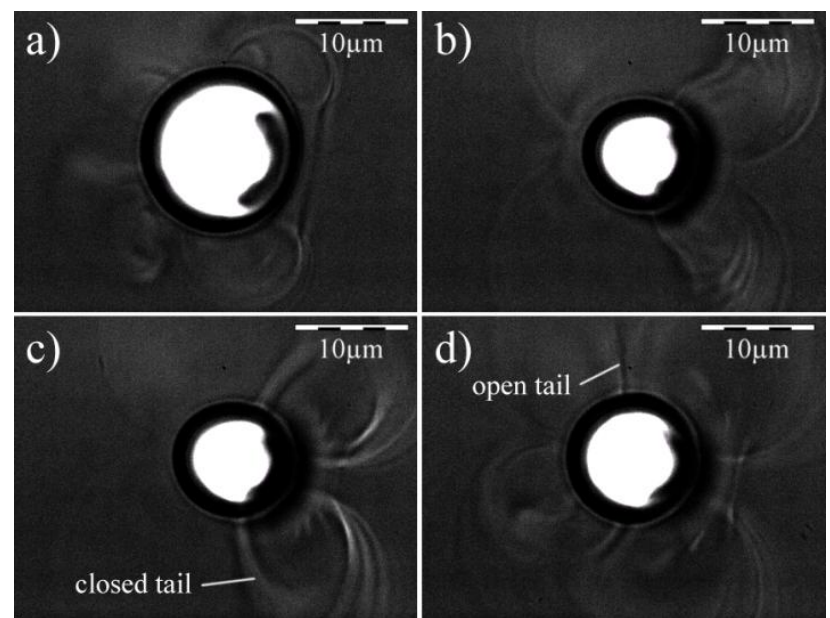

Fig. 3. After flash relaxation: a-b) shrinking of the ring size; c-d) images taken at the end of the second phase. The ring starts to grow (which means, it is already in phase three). Sometimes after ring creation, dark crescent area appears inside it as it is shown in these series of pictures. Generally, it was observed that due to laser radiation, magnetic particles aggregate. For some rings, these aggregates become large and grow inside the ring area.
3. Long term relaxation: This phase could take up to a minute. Through that time, the ring reaches its final size, which is usually about the size it has shortly after the flash. The number of tails does not change during this step, however, sometimes they appear to group on one side of the ring. Moreover, the "big tail" is being created (a strong tail which is brighter than others and almost always opens - Fig. 4) [12]. Interestingly, if there is a dark crescent spot inside the ring, the big tail starts always on the opposite side.

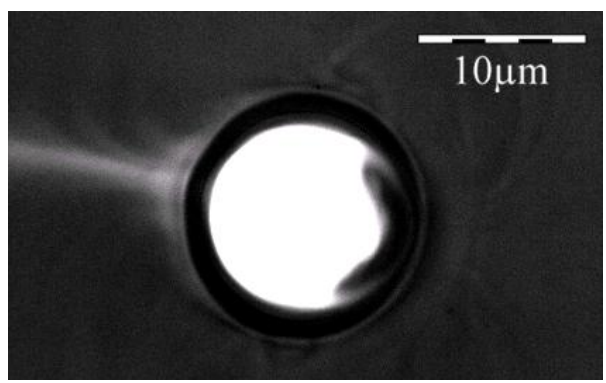

Fig. 4. Long open tail created at the opposite side of the crescent dark spot.

After the ring was formed, it does not remain stable [12]. Its vibrations were observed with a high speed camera (see also movie file 1: ring creation in slow motion - 100× slowed). The frequency of these oscillations is about $350-400 \mathrm{~Hz}$ with amplitude up to single micrometres. Sometimes the orientation of tails is changing with time (Fig. 5).
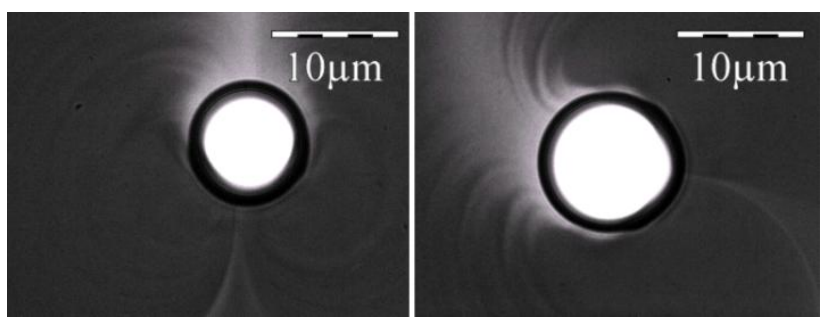

Fig. 5. Reorientation of tails during vibrations of dark ring.

In the mentioned video, it can be observed that closed tails create a space, within which particles of a ferrofluid are moving in circles (along tails, but not only within the tail area) (see movie file 2: ring vibrations in slow motion $-100 \times$ slowed). Two adjacent closed areas rotate always in opposite direction. Moreover, the closed lines seem to grow and disappear (being replaced by others). This behaviour seems to have an impact on the big tail, as the closed tail lines appear to stack in the big tail area, making a bright aura around it (Fig. 6). This process cannot be observed in real time, as the "stacking" is too fast. In a larger time scale, the ring vibrations perform bigger jumps, which may lead to complete reorientation of the tails around it (Fig. 7). Those jumps may even lead to a situation where the dark ring could jump completely out 
of the laser spot. Shortly after such a thing has happened, a new ring is being formed in the optical trap area.

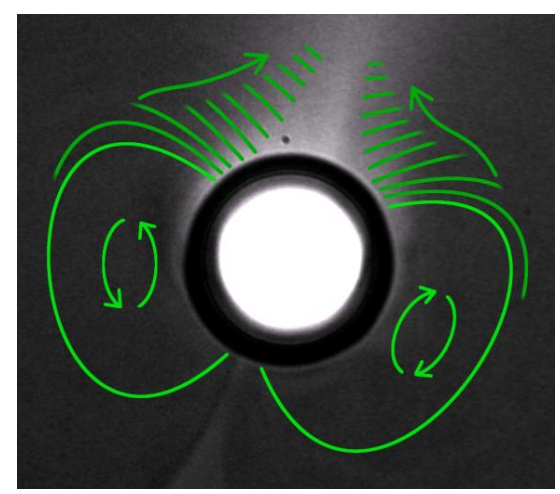

Fig. 6. Illustration of rotating areas and tail stacking. (see also movie file 2 - ring vibrations in slow motion - $100 \times$ slowed).
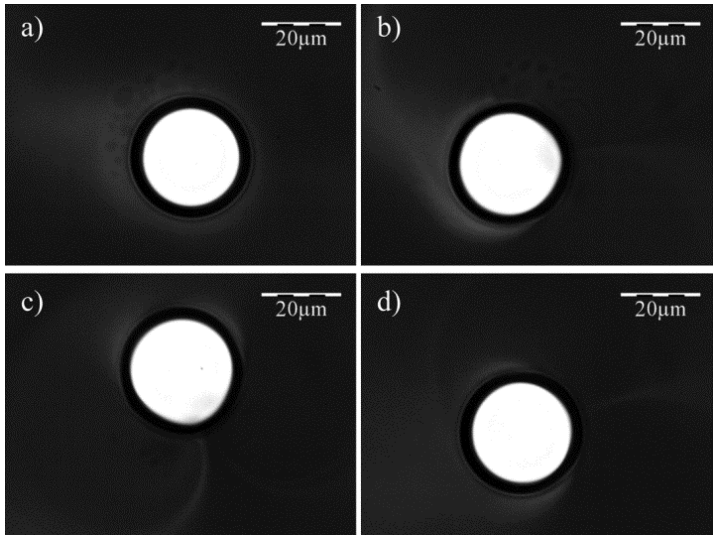

Fig. 7. Hops performed by the dark ring. It stayed in each position for a certain period of time, and then jumped to another (see also movie file 3 - ring hops in real time).
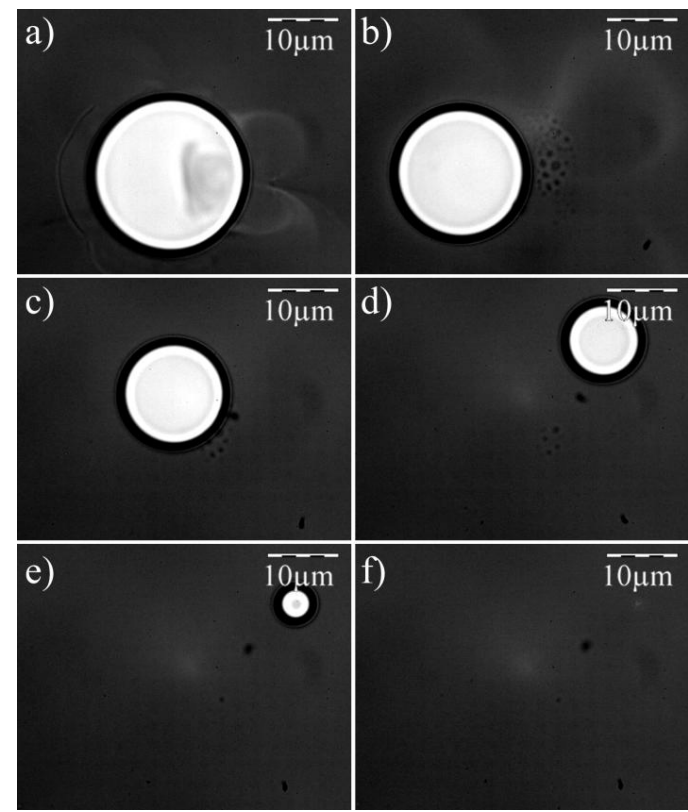

Fig. 8. Switching the laser light off: a) laser is on; $b-f$ ) the laser is switched off. The ring slowly shrinks down and finally disappears.
Because the ring was not kept by the optical trap, it drifted around the sample volume. The time interval between pictures b-f is about $5 \mathrm{~s}$.

When the laser is switched off, the dark rings disappear, but the process is much slower than ring creation. They have a lifetime up to 30s (which depends for example on ferrofluid layer thickness). During this time, the ring slowly shrinks down and finally disappears. Without laser light the tails are not observed. The bright area from the big tail spreads over the whole ring, creating an aura around it. Because the ring is not fixed by an optical trap, it flows out of the observation plane. This flow is smooth and no vibrations or jumps are observed (Fig. 8).

In conclusions, we reported on the new phenomenon in ferrofluids, observed with holographic optical tweezers equipped with a fast video camera. The process of ring creation reported in [12] is very fast, so we could not observe how the ring was developing. The process of ring disappearing is relatively slow and smooth. This shows that although initiated by strong laser radiation the rings have their own stability. They can even escape from the laser beam centre as was shown in Fig. 7 (video 3). Other observed processes are also fast and complicated. The geometry and dynamics of bright tails is strictly related to the flow of magnetic particles observed in a sample volume (Fig. 6).

This work was supported by the National Center for Research and Development (Poland) under grant NR130023-10 and the Polish Ministry of Scientific Research and Information Technology under Grant No. N N518 49883

\section{References}

[1] C. Scherer, M.F. Neto, Brazilian J. Phys. 35, 718 (2005).

[2] J. Philip, J.M. Laskar, J. Nanofluids 1, 3 (2012).

[3] D.G. Grier, Nature 424, 21 (2003).

[4] A. Jonas, P. Zemanek, Electrophoresis 29, 4813 (2008).

[5] E. Curtis, B.A. Koss, D.G. Grier, Opt. Commun. 207, 169 (2002).

[6] K.T. Gahagan, G.A. Swartzlander, Opt. Lett. 21, 827 (1996).

[7] J. Arlt, V. Garcez-Chavez, W. Sibbett, K. Dholakia, Opt. Commun. 197, 239 (2001).

[8] T. Sugiyama, K. Yuyama, H. Masuhara, Accounts Chem. Res. 451946 (2012).

[9] B. Hoffmann, W. Köhler, J. Magn. Magn. Mater. 262, 289 (2003).

[10] R.R. Kellner, W. Köhler, J. Appl. Phys. 97, 034910 (2005).

[11] Zi-Ming Meng et al., J. Appl. Phys. 106, 044905 (2009).

[12] J. Masajada, M. Bacia, S. Drobczyński, Opt. Lett. 383910 (2013). 